\title{
Some New Applications of Elzaki Transform for Solution of Linear Volterra Type Integral Equations
}

\author{
Saad Sharjeel, Mushtaq Ahmed Khan Barakzai \\ College of Computer Science and Information System (CCSIS), Institute of Business Management (IoBM), Karachi, Pakistan \\ Email: std_15459@iobm.edu.pk, mushtaq.barakzai@iobm.edu.pk
}

How to cite this paper: Sharjeel, S. and Barakzai, M.A.K. (2019) Some New Applications of Elzaki Transform for Solution of Linear Volterra Type Integral Equations. Journal of Applied Mathematics and Physics, 7, 1877-1892.

https://doi.org/10.4236/jamp.2019.78129

Received: June 29, 2019

Accepted: August 20, 2019

Published: August 23, 2019

Copyright $\odot 2019$ by author(s) and Scientific Research Publishing Inc. This work is licensed under the Creative Commons Attribution International License (CC BY 4.0).

http://creativecommons.org/licenses/by/4.0/

\begin{abstract}
Volterra type integral equations have diverse applications in scientific and other fields. Modelling physical phenomena by employing integral equations is not a new concept. Similarly, extensive research is underway to find accurate and efficient solution methods for integral equations. Some of noteworthy methods include Adomian Decomposition Method (ADM), Variational Iteration Method (VIM), Method of Successive Approximation (MSA), Galerkin method, Laplace transform method, etc. This research is focused on demonstrating Elzaki transform application for solution of linear Volterra integral equations which include convolution type equations as well as one system of equations. The selected problems are available in literature and have been solved using various analytical, semi-analytical and numerical techniques. Results obtained after application of Elzaki transform have been compared with solutions obtained through other prominent semi-analytic methods i.e. ADM and MSA (limited to first four iterations). The results substantiate that Elzaki transform method is not only a compatible alternate approach to other analytic methods like Laplace transform method but also simple in application once compared with methods ADM and MSA.
\end{abstract}

\section{Keywords}

Elzaki Transform, Linear Volterra Integral Equation, Adomian

Decomposition Method, Method of Successive Approximation,

Laplace Elzaki Duality (LED)

\section{Introduction}

Integral equations find their application in physical sciences, finance, etc. Diffraction problems, water waves, scattering in quantum mechanics are often 
modelled using integral equations [1]. The unknown function which is required to be determined is denoted by $u(x)$ and it appears under the sign of integration. $K(x, t)$ is called "kernel" function whereas $g(x)$ and $h(x)$ are the limits of integration. [1] has provided excellent introduction on Integral equations along with solution methods. General form of an Integral equation can be given as under:

$$
u(x)=f(x)+\lambda \int_{h(x)}^{g(x)} k(x, t) u(t) \mathrm{d} t
$$

An Integro-differential equation is a bit different from Integral equation as it contains unknown function $u(x)$ which appears under integral sign and also has ordinary derivative of unknown function. For the Integro-differential equation, general form can be given as:

$$
u^{n}(x)=f(x)+\lambda \int_{h(x)}^{g(x)} k(x, t) u(t) \mathrm{d} t
$$

The system of integral or Integro-differential equations has two or more equations with two or more variables which are required to be determined. Volterra integral, integro-differential as well as related system of equations contains at least one variable limit of integration. Extensive mathematical techniques are available for finding analytic (exact), approximate analytic as well as numerical solutions of integral equations. Some of the techniques include Adomian Decomposition Method (ADM), Homotopy Perturbation Method (HPM), Homotopy Analysis Method (HAM), Variational Iteration Method (VIE), etc. Transformation methods like Sumudu transform, Laplace transform, etc. are also being used to find solution of integral equations of various types and classes. However, new methods are continuously being explored throughout the world. Elzaki transform has been introduced by [2] and there is a growing interest of researchers in finding various applications of said transformation method. A large number of mathematical problems have been solved using Elzaki transform method. However, with respect to finding its new applications, there is great potential available. Volterra type integral equations have been earlier solved using numerous methods available by mathematicians. In this paper some new applications of Elzaki transform have been discussed to find analytic solution of linear Volterra type integral equations which include convolution type as well as system of equations. The problems selected for demonstrating Elzaki transform application are those which have not been solved earlier using said transformation method. The analytic solutions obtained after application of Elzaki transform have been compared with results obtained through famous semi-analytical methods i.e. ADM and MSA (which have been restricted to first four iterations). The results establish the accuracy and simplicity of Elzaki transform method and also attest its compatibility with Laplace transform.

\section{Literature Review}

Integro-differential equations with bulge function have been examined by [3] Numerical solution has been obtained by applying trapezoidal rule. For finding 
the exact solution; Elzaki and inverse Elzaki transform as well as Taylor series expansion and convolution theorem have been used. Through examples, it has been shown that the approximate solutions acquired by trapezoidal rule are in good agreement with exact solutions obtained through transformation method. [4] conducted comparative study between Adomian Decomposition Method $(\mathrm{ADM})$ and Elzaki transform. Both methods have been used to solve linear partial differential equations with constant coefficients. Elzaki transform method has been used by [5] for solution of systems of linear Integro-differential equations with constant coefficients. Fundamental properties of Elzaki transform have been discussed by [6] and Elzaki transform for comprehensive list of functions has been provided. Furthermore, more general shift theorems have been introduced. Laplace-Elzaki Duality (LED) invoked a complex inverse Elzaki transform, as a Bromwich contour integral formula. [7] researched practical formulae for differentiation of integral transforms used for differential equations with variable coefficients. The transforms which have been checked are Laplace, Sumudu and Elzaki. Moreover, it has been argued that proposed formulae can be applied to almost every equation. [8] proposed shifting theorems for the Elzaki transform to solve initial value problems arising in control engineering. The proposed theorems are composed of $\mathrm{u}$-shifting theorem and time shifting, and the proof is compared with established ones. [9] provided Romberg method for solution of linear Volterra integral equations of second kind. The examples presented in paper show superiority of Romberg method over quadrature method. [10] solved Volterra integral equations with separable kernels using the differential transform method. Approximate solution has been calculated in form of a series with easily calculable terms. Exact solutions of linear as well as nonlinear integral equations have been presented. Results exemplify the reliability of the differential transform method. Collocation method has been presented by [11] for linear Volterra integral equation of the second kind by using Sinc basis functions. Approximate solutions are provided and auxiliary basis functions satisfy four different boundary conditions. Numerical results have been included to confirm efficiency and accuracy of method. [12] solved Volterra integral equations of second kind (convolution type) by using the Elzaki transform. Solution of integro-differential equations using Elzaki transform has been discussed by [13]. Numerical solution of a system of two first order Volterra integro-differential equations arising in ultimate ruin theory has been discussed by [14]. Existence and Uniqueness of Solution of Volterra Integral Equations has been studied by [15]. [16] has used Finite Difference Method for Smooth Solution of Linear Volterra Integral Equations. Numerical Solutions of Volterra Integral Equations Using Galerkin method with Hermite Polynomials have been discussed by [17].

\section{Mathematical Foundations of Elzaki Transform}

Elzaki transform has been derived from classical Fourier Integral.

By definition, Elzaki transform is defined for given set $A$ as: 


$$
A=\left\{f(t)\left|\exists M, k_{1}, k_{2}>0\right| f(t)<M \mathrm{e}^{\frac{-t}{k_{j}}} \text {, if } t \in(-1)^{j} \times[0, \infty)\right\}
$$

For the given function $M$ should be finite however $k_{1}$ and $k_{2}$ may be finite or infinite.

Elzaki transform is denoted by $E($.$) and is given as$

$$
E[f(t)]=T(p)=p \int_{0}^{\infty} f(t) \mathrm{e}^{-t / p} \mathrm{~d} t, t \geq 0, \quad k_{1} \leq p \leq k_{2}
$$

here $T(p)$ is Elzaki transform of integral function $f(t)$.

For $f(t)$ we assume that the integral given in Equation (4) exists.

Let $f(t)=1$

$$
E[1]=T(p)=p \int_{0}^{\infty} 1 \cdot \mathrm{e}^{-t / p} \mathrm{~d} t=p^{2}
$$

Let $f(t)=t$

$$
E[t]=T(p)=p \int_{0}^{\infty} t \cdot \mathrm{e}^{-\frac{t}{p}} \mathrm{~d} t=p^{3}
$$

For the nth order

$$
E\left[t^{n}\right]=n ! p^{n+2}
$$

\subsection{Elzaki Transform of Common Functions}

Elzaki transform of some common functions is given as under Elzaki transform of exponential function

$$
E\left(\mathrm{e}^{a t}\right)=T(p)=p \int_{0}^{\infty} \mathrm{e}^{a t} \cdot \mathrm{e}^{-\frac{t}{p}} \mathrm{~d} t=\frac{p^{2}}{1-a p}
$$

Elzaki transform of Sin function

$$
E(\sin (a t))=\frac{a p^{3}}{a^{2} p^{2}+1}
$$

Elzaki transform of Cosine function

$$
E(\cos (a t))=\frac{p^{2}}{a^{2} p^{2}+1}
$$

Elzaki transform of Sin hyperbolic function

$$
E(\sinh (a t))=\frac{a p^{3}}{1-a^{2} p^{2}}
$$

Similarly Elzaki transform for derivatives of a function can be given as

$$
\begin{gathered}
E\left(f^{\prime}(t)\right)=\frac{T(p)}{p}-v f(0) \\
E\left(f^{\prime \prime}(t)\right)=\frac{T(p)}{p^{2}}-f(0)-v f^{\prime}(0)
\end{gathered}
$$




$$
E\left(f^{n}(t)\right)=\frac{T(p)}{p^{n}}-\sum_{k=0}^{n-1} p^{2-n+k} f^{k}(0)
$$

\subsection{Laplace Elzaki Duality (LED)}

For function $f(t)$ which belongs to set $A$,

$f(t) \in A=\left\{f(t) \mid \exists M, k_{1}, k_{2}>0\right.$, such that $\mid f(t)<M \mathrm{e}^{\frac{|t|}{k_{j}}}$, if $\left.t \in(-1)^{j} \times[0, \infty)\right\}$

Since Laplace transform is given as

$$
F(s)=L(f(t))=\int_{0}^{\infty} \mathrm{e}^{-s t} f(t) \mathrm{d} t
$$

Inter-conversion between Laplace and Elzaki transform can be given by

$$
\begin{aligned}
& T(v)=v F\left(\frac{1}{v}\right) \\
& F(s)=s T\left(\frac{1}{s}\right)
\end{aligned}
$$

\section{Examples}

\section{Example 4.1}

$$
u(x)=4 x+2 x^{2}-\int_{0}^{x} u(t) \mathrm{d} t
$$

This problem has been taken from [18].

Taking Elzaki transform on both sides of Equation (19)

$$
\begin{gathered}
E[u(x)]=4[E(x)]+2\left[E\left(x^{2}\right)\right]-E\left[\int_{0}^{x} u(t) \mathrm{d} t\right] \\
T[v]=4 v^{3}+2\left(2 ! v^{2+2}\right)-v T[v] \\
T[v]=4 v^{3}+4 v^{4}-v T[v] \\
T[v]+v T[v]=4\left(v^{3}+v^{4}\right) \\
T[v]=\frac{4 v^{3}(1+v)}{1+v}
\end{gathered}
$$

After simplification,

$$
T[v]=4 v^{3}
$$

Taking Inverse Elzaki transform on both sides of Equation (25), we get,

$$
u(x)=4 x
$$

Hence, required analytic solution is obtained.

\section{Example 4.2}

$$
u(x)=x^{2}+\frac{1}{12} x^{4}+\int_{0}^{x}((t-x) u(t)) \mathrm{d} t
$$

This problem has been discussed by [18]. 
Taking derivatives and applying Leibniz rule to equation Equation (27)

$$
\begin{gathered}
u^{\prime}(x)=2 x+\frac{1}{3} x^{3}-\int_{0}^{x} u(t) \mathrm{d} t \\
u^{\prime \prime}(x)=2+x^{2}-u(x) \\
u^{\prime \prime}(x)+u(x)=2+x^{2}, \text { with } u(0)=0, u^{\prime}(0)=0
\end{gathered}
$$

Since,

$$
E\left[u^{\prime \prime}(x)\right]=\frac{T[v]}{v^{2}}-u(0)-v u^{\prime}(0)
$$

After plugging initial values we obtain,

$$
E\left[u^{\prime \prime}(x)\right]=\frac{T[v]}{v^{2}}
$$

Hence, the Elzaki transform of given equation will be

$$
E\left[u^{\prime \prime}(x)\right]+E[u(x)]=E[2]+E\left[x^{2}\right]
$$

Or,

$$
\begin{gathered}
\frac{T[v]}{v}+T[v]=2+2 v^{4} \\
T[v]+v^{2} T[v]=2 v^{2}\left(v^{2}+v^{4}\right) \\
T[v]=\frac{2 v^{2}\left(v^{2}+v^{4}\right)}{1+v^{2}}
\end{gathered}
$$

Taking inverse Elzaki transform on both sides of Equation (36) we get,

$$
\begin{gathered}
u(x)=E^{-1}\left[\frac{2 v^{2}\left(v^{2}+v^{4}\right)}{1+v^{2}}\right] \\
u(x)=E^{-1}\left[2 v^{4}\right] \\
u(x)=x^{2}
\end{gathered}
$$

Hence, required analytic solution is obtained.

\section{Example 4.3}

Consider Volterra Integral equation of Convolution type:

$$
u(x)=2+\int_{0}^{x}(x-t) u(t) \mathrm{d} t
$$

This problem has been taken from [18],

Taking Elzaki transform on both sides of Equation (40),

$$
\begin{gathered}
E[u(x)]=E[2]+E\left[\int_{0}^{x}((x-t) u(t)) \mathrm{d} t\right] \\
T[v]=2 v^{2}+\frac{1}{v}\left[v^{3} T[v]\right] \\
T[v]=2 v^{2}+v^{2} T[v]
\end{gathered}
$$




$$
\begin{gathered}
T[v]-v^{2} T[v]=2 v^{2} \\
T[v]=\frac{2 v^{2}}{1-v^{2}}
\end{gathered}
$$

Taking Inverse Elzaki transform on both sides of Equation (45),

$$
u(x)=2 \cosh x
$$

Hence, required analytic solution is obtained.

\section{Example 4.4}

This problem has been solved by [11] using Sinc basis functions,

$$
u(x)=x-x^{2}+\frac{x^{3}}{6}-\frac{x^{4}}{12}+\int_{0}^{x}((t-x) u(t)) \mathrm{d} t, 0 \leq x \leq 1
$$

Taking Elzaki transform on both sides of Equation (47),

$$
\begin{gathered}
E[u]=E[x]-E\left[x^{2}\right]+\frac{1}{6} E\left[x^{3}\right]-\frac{1}{12} E\left[x^{4}\right]+E\left[\int_{0}^{x}((t-x) u(t)) \mathrm{d} t\right] \\
T[v]=v^{3}-2 v^{4}+v^{5}-2 v^{6}-\frac{1}{v}(E[t] E[u]) \\
T[v]=v^{3}-2 v^{4}+v^{5}-2 v^{6}-v^{2} T[v] \\
T[v]=v^{3}-2 v^{4}
\end{gathered}
$$

Taking Inverse Elzaki transform on both sides,

$$
u(x)=x-x^{2}
$$

Hence, required analytic solution is obtained.

\section{Example 4.5}

Consider convolution type linear Volterra integral equation. [10] solved the problem using Differential Transform Method (DTM),

$$
u(x)=1-x-\frac{x^{2}}{2}+\int_{0}^{x}((x-t) u(t)) \mathrm{d} t, 0<x<1
$$

Taking Elzaki transform on both sides of Equation (53),

$$
\begin{gathered}
E[u(x)]=E[1]-E[x]-E\left[\frac{x^{2}}{2}\right]+E\left[\int_{0}^{x}((x-t) u(t)) \mathrm{d} t\right] \\
T[v]=v^{2}-v^{3}-v^{4}+\frac{1}{v}[E[t] E[u]] \\
T[v]=v^{2}-v^{3}-v^{4}+v^{2} T[v] \\
T[v]=\frac{v^{2}-v^{3}-v^{4}}{1-v^{2}}
\end{gathered}
$$

Through Taylor expansion about $v=0$,

$$
T[v]=v^{2}-v^{3}-v^{5}-v^{7}-v^{9}-\cdots
$$

Taking Inverse Elzaki transform on both sides of Equation (58), 


$$
E^{-1}[T[v]]=E^{-1}\left[v^{2}\right]-E^{-1}\left[v^{3}\right]-E^{-1}\left[v^{5}\right]-E^{-1}\left[v^{7}\right]-\cdots
$$

In series form,

$$
u(x)=1-\left(x+\frac{x^{3}}{6}+\frac{x^{5}}{120}+\frac{x^{7}}{5040}+\frac{x^{9}}{362880}+O\left(x^{11}\right)\right)
$$

In closed form,

$$
u(x)=1-\sinh (x)
$$

Similarly we can also use LED and demonstrate that it gives same result as obtained using Taylor expansion. Since,

$$
F(s)=s T\left(\frac{1}{s}\right)
$$

We can write Equation as,

$$
\begin{gathered}
F(s)=\mathcal{L}_{s}^{-1}\left[\frac{s\left(-\left(\frac{1}{s}\right)^{4}-\left(\frac{1}{s}\right)^{3}+\left(\frac{1}{s}\right)^{2}\right)}{1-\left(\frac{1}{s}\right)^{2}}\right](x) \\
u(x)=\frac{\mathrm{e}^{-x}}{2}-\frac{\mathrm{e}^{x}}{2}+1
\end{gathered}
$$

Writing in closed form,

$$
u(x)=1-\sinh (x)
$$

Hence, required analytic solution is obtained.

\section{Example 4.6}

This system of equations has been solved by [19] using rationalized Haar functions.

$$
\begin{gathered}
y_{1}(t)-\int_{0}^{t} y_{2}(s) \mathrm{d} s=1-t^{2} \\
y_{2}(t)-\int_{0}^{t} y_{1}(s) \mathrm{d} s=t
\end{gathered}
$$

Take Elzaki transform on both sides of Equation (64) we have,

$$
\begin{gathered}
E\left[y_{1}(t)\right]-E\left[\int_{0}^{t} y_{2}(s) \mathrm{d} s\right]=E[1]-E\left[t^{2}\right] \\
\bar{y}_{1}[v]-v \bar{y}_{2}[v]=v^{2}-2 v^{4}
\end{gathered}
$$

Similarly take Elzaki transform of second equation i.e. Equation (65),

$$
\begin{gathered}
E\left[y_{2}(t)\right]-E\left[\int_{0}^{t} y_{1}(s) \mathrm{d} s\right]=E[t] \\
\bar{y}_{2}[v]-v \bar{y}_{1}[v]=v^{3}
\end{gathered}
$$

After simplification,

$$
\bar{y}_{2}[v]=2 v^{3}
$$

Take Inverse Elzaki transform of Equation (70) we have, 


$$
\begin{gathered}
E^{-1}\left[\bar{y}_{2}[v]\right]=E^{-1}\left[2 v^{3}\right] \\
y_{2}(t)=2 t
\end{gathered}
$$

And from Equation (69) we have,

$$
\begin{gathered}
\bar{y}_{2}[v]-v \bar{y}_{1}[v]=v^{3} \\
\bar{y}_{1}[v]=v^{2}
\end{gathered}
$$

Take Inverse Elzaki transform of Equation (73) we have,

$$
\begin{gathered}
E^{-1}\left[\bar{y}_{1}[v]\right]=E^{-1}\left[v^{2}\right] \\
y_{1}(t)=1
\end{gathered}
$$

Equation (72) and Equation (75) provides analytic solution.

\section{Results and Discussions}

It has been established that Elzaki transform can be easily implemented to find analytic solution of Volterra type integral equations as discussed through various problems in section 4. In this section we shall provide the solution for domain 0 to 1 and for comparison purposes, semi-analytic methods (ADM and MSA with zero as initial guess) solutions (limited to first four iterations), have also been given. It is likely that results of semi-analytic methods will converge to analytic solution if more iteration is carried out; but same will be at cost of increased computational work. All computational work has been carried out in Mathematica version 9 .

For Example 4.1 the analytic solution is $u(x)=4 x$ which has also been achieved through application of Elzaki transform while the error for ADM and MSA once restricted to the first four iterations is evident (Table 1). However, ADM in this case produced better results once compared with MSA. Significant deviation of MSA from analytic results is obvious in Figure 1.

For Example 4.2 the analytic solution is $u(x)=x^{2}$ which has also been achieved through application of Elzaki transform while the error for ADM and MSA once restricted to first four iterations is apparent (Table 2). However, ADM in this case has much better results as compared to MSA. MSA is showing large deviation from analytic results (Figure 2).

For Example 4.3 the analytic solution is $u(x)=2 \cosh x$ which has also been achieved through application of Elzaki transform. ADM has slightly better accuracy once compared with MSA. Table 3 and Figure 3 depict results in numeric and graphical form respectively.

For Example 4.4 the analytic solution is $u(x)=x-x^{2}$ which has also been achieved through application of Elzaki transform while the error for ADM and MSA once restricted to first four iterations is shown in Table 4. Figure 4 depicts results in graphical form.

Graph plot shows considerable variation of results of MSA from analytic result. 
Table 1. Solution of Example 4.1 using different methods and absolute errors for approximate analytic methods.

\begin{tabular}{cccccc}
\hline $\boldsymbol{x}$ & Analytic & ADM & MSA & Abs Error MSA Abs Error ADM \\
\hline 0 & 0.000000 & 0.000000 & 0.000000 & 0.000000 & 0.000000 \\
0.1 & 0.400000 & 0.400000 & 0.400183 & 0.000183 & 0.000000 \\
0.2 & 0.800000 & 0.799989 & 0.801600 & 0.001600 & 0.000011 \\
0.3 & 1.200000 & 1.199919 & 1.205850 & 0.005850 & 0.000081 \\
0.4 & 1.600000 & 1.599659 & 1.614933 & 0.014933 & 0.000341 \\
0.5 & 2.000000 & 1.998958 & 2.031250 & 0.031250 & 0.001042 \\
0.6 & 2.400000 & 2.397408 & 2.457600 & 0.057600 & 0.002592 \\
0.7 & 2.800000 & 2.794398 & 2.897183 & 0.097183 & 0.005602 \\
0.8 & 3.200000 & 3.189077 & 3.353600 & 0.153600 & 0.010923 \\
0.9 & 3.600000 & 3.580317 & 3.830850 & 0.230850 & 0.019683 \\
1 & 4.000000 & 3.966667 & 4.333333 & 0.333333 & 0.033333 \\
\hline
\end{tabular}

Table 2. Solution of Example 4.2 using different methods and absolute errors for approximate analytic methods.

\begin{tabular}{cccccc}
\hline $\boldsymbol{x}$ & Analytic & ADM & MSA & Abs Error ADM Abs Error MSA \\
\hline 0 & 0.000000 & 0.000000 & 0.000000 & 0.000000 & 0.000000 \\
0.1 & 0.010000 & 0.010000 & 0.009958 & 0.000000 & 0.000042 \\
0.2 & 0.040000 & 0.040002 & 0.039333 & 0.000002 & 0.000667 \\
0.3 & 0.090000 & 0.090027 & 0.086625 & 0.000027 & 0.003375 \\
0.4 & 0.160000 & 0.160146 & 0.149334 & 0.000146 & 0.010666 \\
0.5 & 0.250000 & 0.250531 & 0.223964 & 0.000531 & 0.026036 \\
0.6 & 0.360000 & 0.361484 & 0.306023 & 0.001484 & 0.053977 \\
0.7 & 0.490000 & 0.493435 & 0.390038 & 0.003435 & 0.099962 \\
0.8 & 0.640000 & 0.646858 & 0.469566 & 0.006858 & 0.170434 \\
0.9 & 0.810000 & 0.822055 & 0.537223 & 0.012055 & 0.272777 \\
1 & 1.000000 & 1.018750 & 0.584722 & 0.018750 & 0.415278 \\
\hline
\end{tabular}

Table 3. Solution of Example 4.3 using different methods and absolute errors.

\begin{tabular}{cccccc}
\hline $\boldsymbol{x}$ & Analytic & ADM & MSA & Abs Error ADM & Abs Error MSA \\
\hline 0 & 2.000000 & 2.000000 & 2.000000 & 0.000000 & 0.000000 \\
0.1 & 2.010008 & 2.010008 & 2.010008 & 0.000000 & 0.000000 \\
0.2 & 2.040134 & 2.040134 & 2.040133 & 0.000000 & 0.000000 \\
0.3 & 2.090677 & 2.090677 & 2.090675 & 0.000000 & 0.000002 \\
0.4 & 2.162145 & 2.162145 & 2.162133 & 0.000000 & 0.000011 \\
0.5 & 2.255252 & 2.255252 & 2.255208 & 0.000000 & 0.000044 \\
0.6 & 2.370930 & 2.370930 & 2.370800 & 0.000001 & 0.000130 \\
0.7 & 2.510338 & 2.510335 & 2.510008 & 0.000003 & 0.000330 \\
0.8 & 2.674870 & 2.674862 & 2.674133 & 0.000008 & 0.000737 \\
0.9 & 2.866173 & 2.866151 & 2.864675 & 0.000022 & 0.001498 \\
1 & 3.086161 & 3.086111 & 3.083333 & 0.000050 & 0.002828 \\
\hline
\end{tabular}


Table 4. Solution of Example 4.4 using different methods and absolute errors.

\begin{tabular}{cccccc}
\hline $\boldsymbol{x}$ & Analytic & ADM & MSA & Abs Error ADM & Abs Error MSA \\
\hline 0 & 0.000000 & 0.000000 & 0.000000 & 0.000000 & 0.000000 \\
0.1 & 0.090000 & 0.090001 & 0.089708 & 0.000001 & 0.000292 \\
0.2 & 0.160000 & 0.160021 & 0.158000 & 0.000021 & 0.002000 \\
0.3 & 0.210000 & 0.210147 & 0.204376 & 0.000147 & 0.005624 \\
0.4 & 0.240000 & 0.240560 & 0.229339 & 0.000560 & 0.010661 \\
0.5 & 0.250000 & 0.251516 & 0.234402 & 0.001516 & 0.015598 \\
0.6 & 0.240000 & 0.243278 & 0.222093 & 0.003278 & 0.017907 \\
0.7 & 0.210000 & 0.215998 & 0.195971 & 0.005998 & 0.014029 \\
0.8 & 0.160000 & 0.169574 & 0.160641 & 0.009574 & 0.000641 \\
0.9 & 0.090000 & 0.103489 & 0.121770 & 0.013489 & 0.031770 \\
1 & 0.000000 & 0.016667 & 0.086111 & 0.016667 & 0.086111 \\
\hline
\end{tabular}

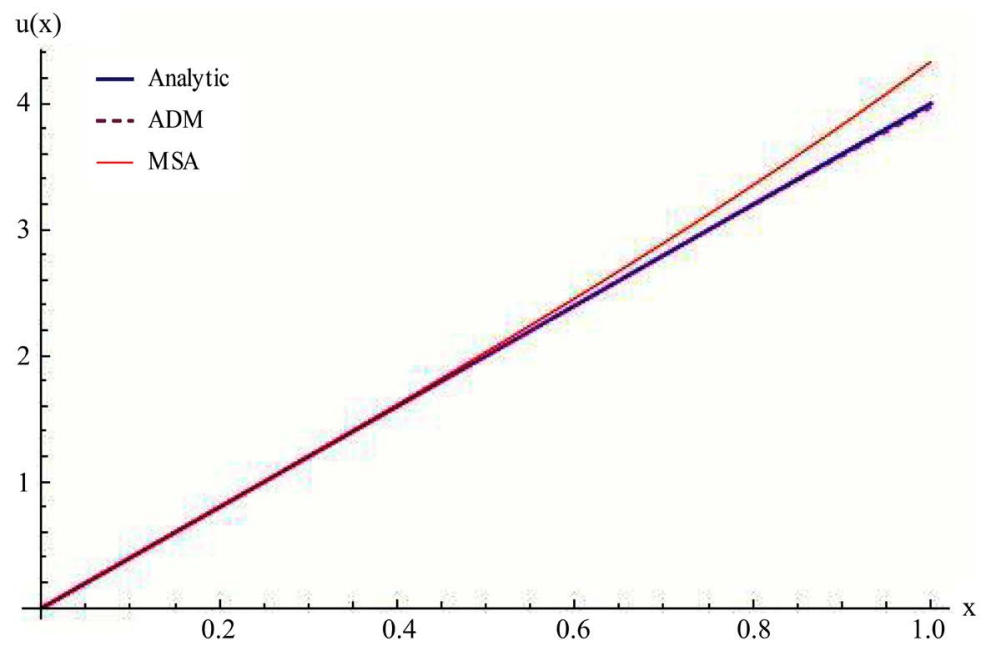

Figure 1. Comparison graph of solution for Example 4.1.

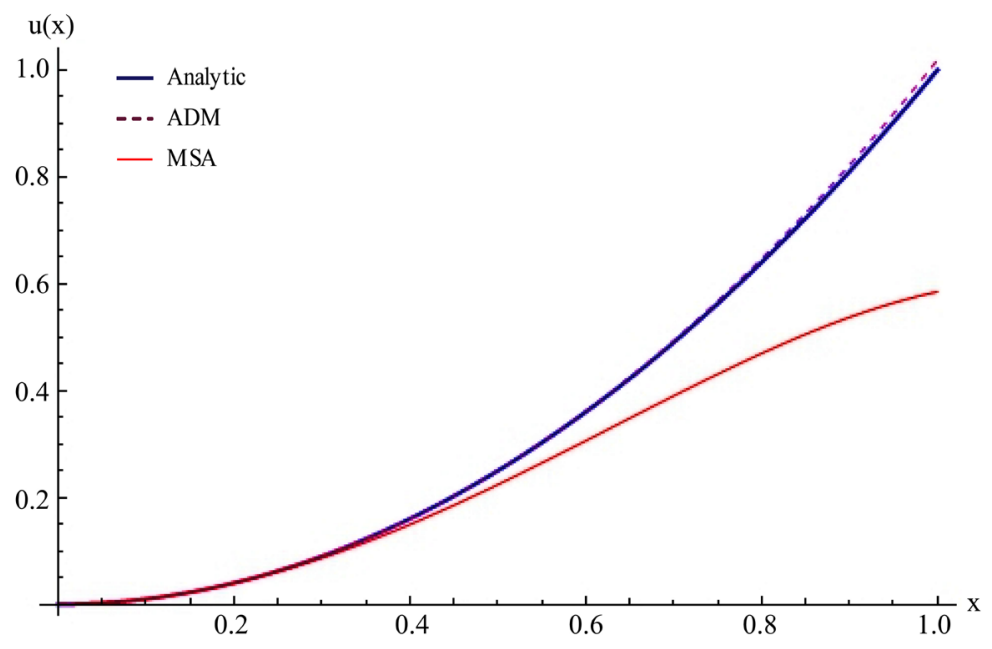

Figure 2. Comparison graph of solution for Example 4.2. 


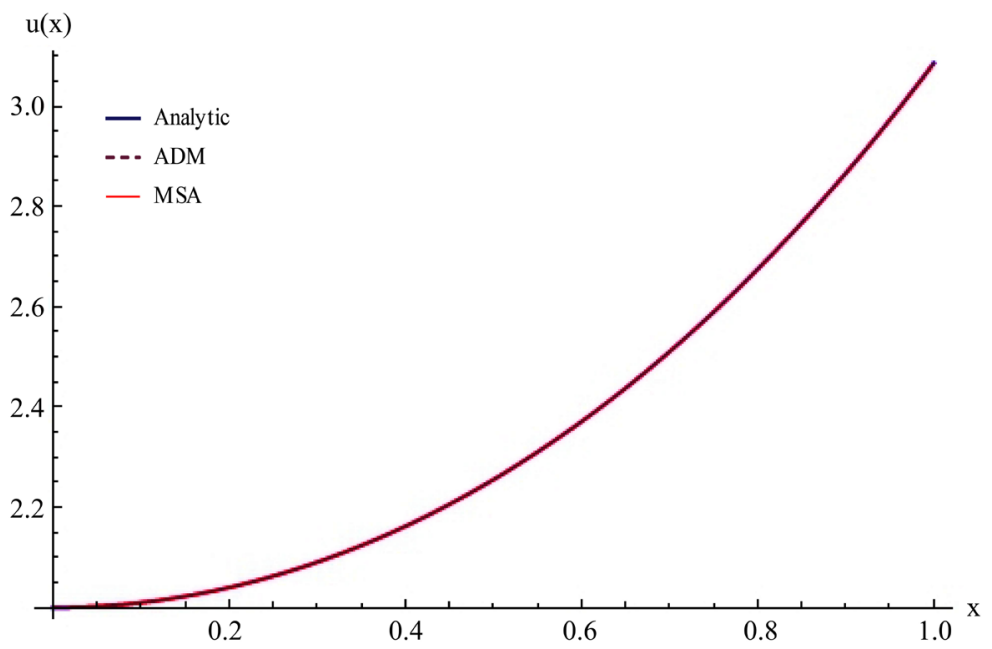

Figure 3. Comparison graph of solution for Example 4.3.

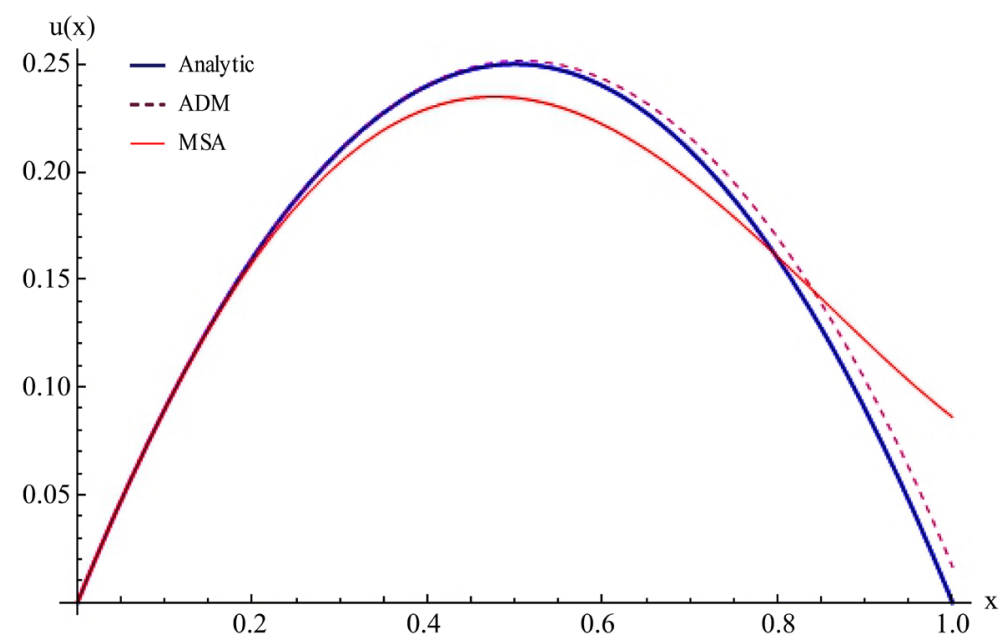

Figure 4. Comparison graph of solution for Example 4.4.

For Example 4.5 the analytic solution is $u(x)=1-\sinh (x)$ which has also been achieved through application of Elzaki transform while the error for ADM and MSA once restricted to first four iterations is evident (Table 5). Figure 5 is showing graphical comparison between methods.

Deviation of MSA solution from analytic result is clearly evident.

For Example 4.6 one analytic solution is $u(t)=y_{1}(t)=1$ which has also been achieved through application of Elzaki transform. ADM and MSA are showing large deviation from analytic result. Results have been depicted in Table 6(a) while comparison graph between methods has been illustrated at Figure 6(a).

$\mathrm{ADM}$ and MSA are showing large deviation from analytic result.

For example 4.6 the second analytic solution is $v(t)=y_{2}(t)=2 t$ which has also been achieved through application of Elzaki transform while the error for ADM and MSA once restricted to first four iterations is obvious. Table 6(b) provides results in numeric form while comparison plot between methods has been given at Figure 6(b). 
Table 5. Solution of Example 4.5 using different methods and absolute errors.

\begin{tabular}{cccccc}
\hline $\boldsymbol{x}$ & Analytic & ADM & MSA & Abs Error ADM Abs Error MSA \\
\hline 0 & 1.000000 & 1.000000 & 1.000000 & 0.000000 & 0.000000 \\
0.1 & 0.899833 & 0.899833 & 0.899499 & 0.000000 & 0.000334 \\
0.2 & 0.798664 & 0.798664 & 0.795972 & 0.000000 & 0.002692 \\
0.3 & 0.695480 & 0.695480 & 0.686282 & 0.000000 & 0.009197 \\
0.4 & 0.589248 & 0.589248 & 0.567061 & 0.000000 & 0.022186 \\
0.5 & 0.478905 & 0.478905 & 0.434570 & 0.000000 & 0.044334 \\
0.6 & 0.363346 & 0.363346 & 0.284548 & 0.000000 & 0.078798 \\
0.7 & 0.241416 & 0.241415 & 0.112043 & 0.000001 & 0.129373 \\
0.8 & 0.111894 & 0.111890 & -0.088768 & 0.000004 & 0.200662 \\
0.9 & -0.026517 & -0.026526 & -0.324779 & 0.000010 & 0.298262 \\
1 & -0.175201 & -0.175223 & -0.604167 & 0.000022 & 0.428965 \\
\hline
\end{tabular}

Table 6. (a) Solution of Example 4.6 using different methods and absolute errors for $u(t)=y_{1}(t)$. (b) Solution of Example 4.6 using different methods and absolute errors for $v(t)=y_{2}(t)$.

(a)

\begin{tabular}{cccccc}
\hline $\boldsymbol{x}$ & Analytic & ADM & MSA & Abs Error ADM & Abs Error MSA \\
\hline 0 & 1.000000 & 1.000000 & 1.000000 & 0.000000 & 0.000000 \\
0.1 & 1.000000 & 1.000158 & 0.999992 & 0.000158 & 0.000008 \\
0.2 & 1.000000 & 1.001195 & 0.999867 & 0.001195 & 0.000133 \\
0.3 & 1.000000 & 1.003785 & 0.999325 & 0.003784 & 0.000675 \\
0.4 & 1.000000 & 1.008363 & 0.997867 & 0.008363 & 0.002133 \\
0.5 & 1.000000 & 1.015104 & 0.994792 & 0.015104 & 0.005208 \\
0.6 & 1.000000 & 1.023904 & 0.989200 & 0.023904 & 0.010800 \\
0.7 & 1.000000 & 1.034357 & 0.979992 & 0.034357 & 0.020008 \\
0.8 & 1.000000 & 1.045739 & 0.965867 & 0.045739 & 0.034133 \\
0.9 & 1.000000 & 1.056984 & 0.945325 & 0.056983 & 0.054675 \\
1 & 1.000000 & 1.066667 & 0.916667 & 0.066667 & 0.083333 \\
\hline
\end{tabular}

(b)

\begin{tabular}{cccccc}
\hline $\boldsymbol{x}$ & Analytic & ADM & MSA & Abs Error ADM & Abs Error MSA \\
\hline 0 & 0.000000 & 0.000000 & 0 & 0.000000 & 0.000000 \\
0.1 & 0.200000 & 0.200000 & 0.199833 & 0.000000 & 0.000167 \\
0.2 & 0.400000 & 0.399995 & 0.398667 & 0.000005 & 0.001333 \\
0.3 & 0.600000 & 0.599960 & 0.5955 & 0.000040 & 0.004500 \\
0.4 & 0.800000 & 0.799829 & 0.789333 & 0.000171 & 0.010667 \\
0.5 & 1.000000 & 0.999479 & 0.979167 & 0.000521 & 0.020833 \\
0.6 & 1.200000 & 1.198704 & 1.164 & 0.001296 & 0.036000 \\
0.7 & 1.400000 & 1.397199 & 1.342833 & 0.002801 & 0.057167 \\
0.8 & 1.600000 & 1.594539 & 1.514667 & 0.005461 & 0.085333 \\
0.9 & 1.800000 & 1.790159 & 1.6785 & 0.009842 & 0.121500 \\
1 & 2.000000 & 1.983333 & 1.833333 & 0.016667 & 0.166667 \\
\hline
\end{tabular}




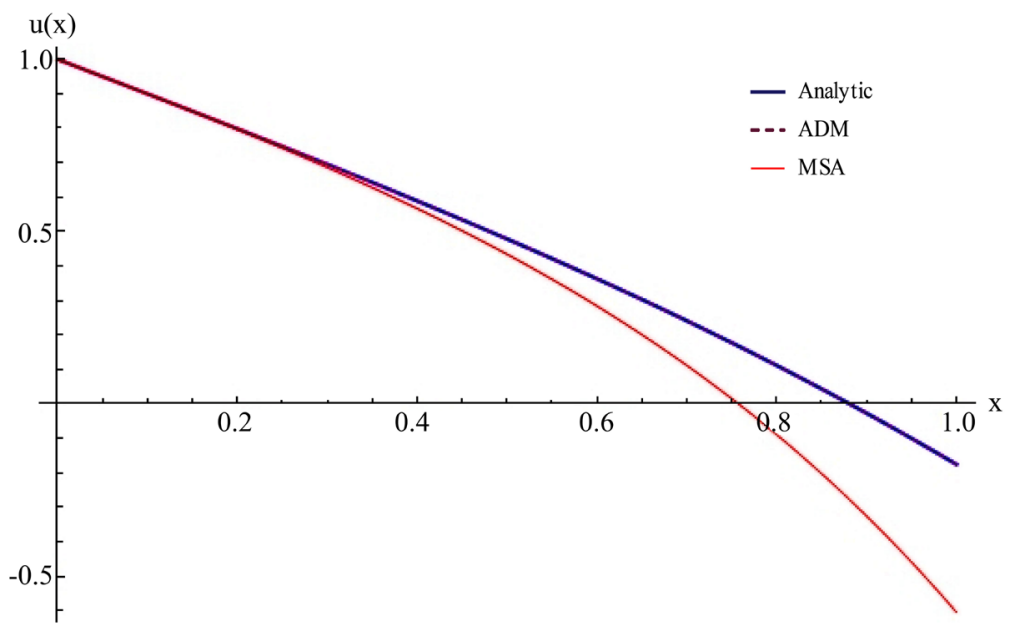

Figure 5. Comparison graph of solution for Example 4.5.

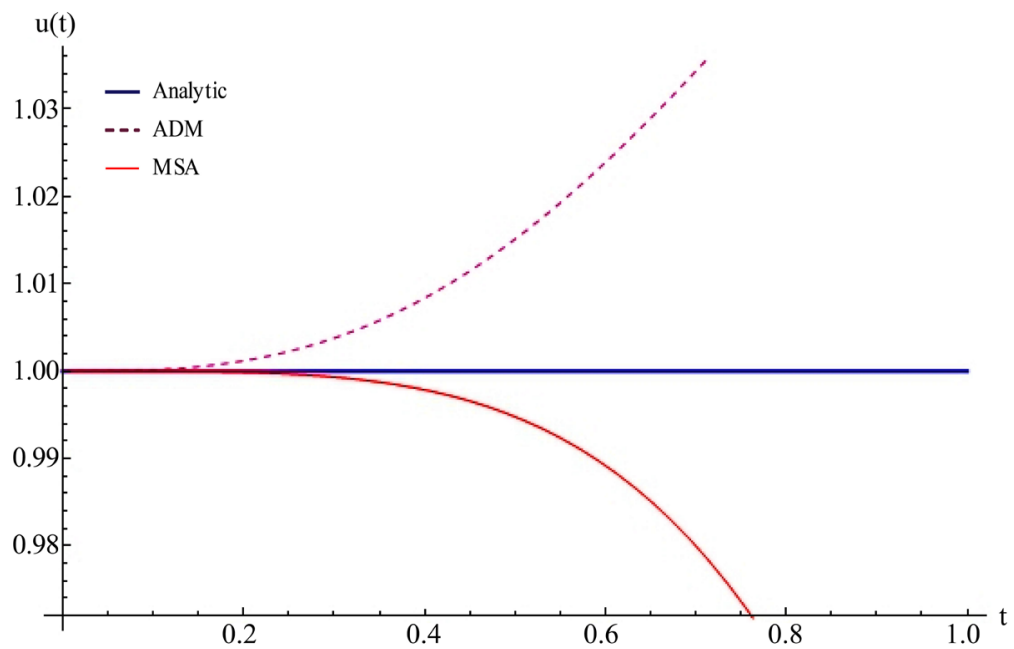

(a)

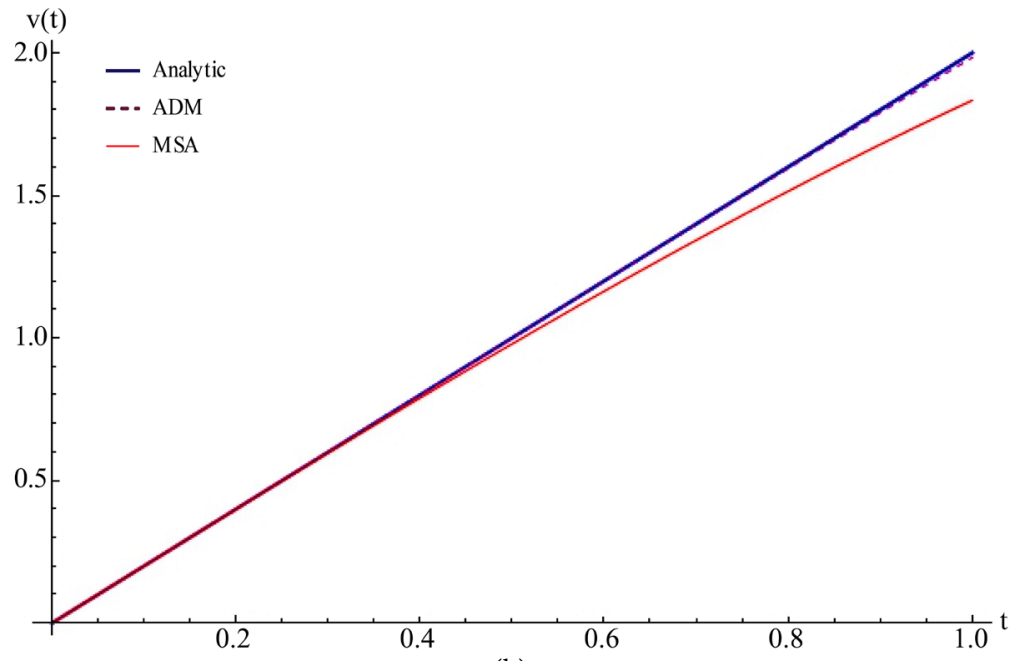

(b)

Figure 6. (a). Comparison graph of solution for example 4.6 (for $u(t)=y_{1}(t)$ );

(b) Comparison graph of solution for Example 4.6 (for $v(t)=y_{2}(t)$ ). 
ADM has considerably good accuracy once compared with MSA.

Accurate analytic solution achievement is useful advantage of Elzaki transform. Moreover, the application process is very simple. Methods like ADM despite having extensive applications and advantages, has not been able to produce analytic results for problems once restricted to fewer iterations (in this case four iterations). MSA further produced results of lower accuracy once compared with ADM.

\section{Conclusion}

In this research Elzaki transform has been successfully applied to linear Volterra type integral equations to find analytic solutions. It has been established that Elzaki transform is a robust compatible alternative to other well-known analytic methods. Moreover, in comparison to notable semi/approximate analytic methods like Adomian Decomposition Method and Method of Successive Approximations, it is not only accurate but often easier to apply. Results presented in research substantiate this claim. The research can be further extended by discussing application of Elzaki transform for linear Volterra type integral equations with separable kernels.

\section{Acknowledgements}

The authors are grateful to the Dr. Ejaz Ahmed and Dr. Aqil Burney for their valuable comments and suggestions on the original manuscript.

\section{Conflicts of Interest}

The authors declare no conflicts of interest regarding the publication of this paper.

\section{References}

[1] Wazwaz, A.-M. (2011) Linear and Nonlinear Integral Equations: Methods and Applications. Higher Education Press, Beijing. https://doi.org/10.1007/978-3-642-21449-3

[2] Elzaki, T.M. (2011) The New Integral Transform "Elzaki Transform". Global Journal of Pure and Applied Mathematics, 7, 57-64.

[3] Abdelrahim Mahgob, M.M. and Elzaki, T.M. (2017) Elzaki Transform and Integro-Differential Equation with a Bulge Function. IOSR Journal of Mathematics, 11, 2278-5728.

[4] Adam, B.A.A. (2015) A Comparative Study of Adomain Decomposition Method and the New Integral Transform "Elzaki Transform". International Journal of Applied Mathematical Research, 4, 8-14. https://doi.org/10.14419/ijamr.v4i1.3799

[5] Elzaki, T.M. and Ezaki, S.M. (2011) On the Solution of Integro-Differential Equation Systems by Using ELzaki Transform. Global Journal of Mathematical Science: Theory and Practical, 3, 13-23. http://www.irphouse.com

[6] Elzaki, T.M., Elzaki, S.M. and Elnour, E.A. (2012) On the New Integral Transform "ELzaki Transform" Fundamental Properties Investigations and Applications. Global Journal of Mathematical Science: Theory and Practical, 4, 15-23. 
[7] Jung, K. and Kim, H. (2014) The Practical Formulas for Differentiation of Integral Transforms. International Journal of Mathematical Analysis, 8, 471-480. https://doi.org/10.12988/ijma.2014.4238

[8] Kim, H. (2014) A Note on the Shifting Theorems for the Elzaki Transform. International Journal of Mathematical Analysis, 8, 481-488.

https://doi.org/10.12988/ijma.2014.4248

[9] Ocvirk, E. (2007) An Application of Romberg Extrapolation on Quadrature Method for Solving Linear Volterra Integral Equations of the Second Kind. Applied Mathematics and Computation, 194, 389-393. https://doi.org/10.1016/j.amc.2007.04.043

[10] Odibat, Z.M. (2008) Differential Transform Method for Solving Volterra Integral Equation with Separable Kernels. Mathematical and Computer Modelling, 48, 1144-1149. https://doi.org/10.1016/j.mcm.2007.12.022

[11] Rashidinia, J. and Zarebnia, M. (2007) Solution of a Volterra Integral Equation by the Sinc-Collocation Method. Journal of Computational and Applied Mathematics, 206, 801-813. https://doi.org/10.1016/j.cam.2006.08.036

[12] Song, Y. and Kim, H. (2014) The Solution of Volterra Integral Equation of the Second Kind by Using the Elzaki Transform. Applied Mathematical Sciences, 8, 525-530. https://doi.org/10.12988/ams.2014.312715

[13] Elzaki, T.M. and Ezaki, S.M. (2011) Solution of Integro-Differential Equations by Using ELzaki Transform. Global Journal of Mathematical Science: Theory and Practical, 3, 1-11. http://www.irphouse.com

[14] Makroglou, A. and Konstantinides, D. (2006) Numerical Solution of a System of Two First Order Volterra Integro-Differential Equations Arising in Ultimate Ruin Theory.

[15] Khezerloo, M. (2012) Existence and Uniqueness of Solution of Volterra Integral Equations. International Journal of Industrial Mathematics, 4, 69-76.

[16] Jalalvand, M., Jazbi, B. and Mokhtarzadeh, M.R. (2013) A Finite Difference Method for the Smooth Solution of Linear Volterra Integral Equations. The International Journal of Nonlinear Analysis and Applications (IJNAA), 4, 1-10.

[17] Rahman, M.M. (2013) Numerical Solutions of Volterra Integral Equations Using Galerkin Method with Hermite Polynomials. Proceedings of the 2013 International Conference on Applied Mathematics and Computational Methods in Engineering, Rhodes Island, 16-19 July 2013, 276-281.

[18] Wazwaz, A.-M. (2015) A First Course in Integral Equations. Second Edition, World Scientific Publishing Company, Singapore. https://doi.org/10.1016/j.jneuroim.2016.09.018

[19] Mirzaee, F. (2010) Numerical Computational Solution of the Linear Volterra Integral Equations System via Rationalized Haar Functions. Journal of King Saud University-Science, 22, 265-268. https://doi.org/10.1016/j.jksus.2010.05.010 